\title{
О ВОСПРИИМЧИВОСТИ ЦТМП ПРИ ПЕРЕМАГНИЧИВАНИИ вдоль Отн
}

Цилиндрические тонкие магнитные пленки (ЦТМП) с неразрушающим считыванием информации, используемые в элементах памяти, представляют большой практический интерес. Технологически вопрос получения таких ЦТМП решен путем создания определенной шероховатости на поверхности подложки, а для улучшения свойств неразрушающего считывания на такую подложку осаждают не один, а несколько магнитных слоев с разной коэрцитивностью. Теоретическое же объяснение связи между шероховатостью и неразрушающими свойствами ЦТМП пока отсутствует.

В настоящей работе делается попытка объяснить процесс неразрушающего считывания путем введения в теорию микромагнетизма двумерных магнитных слоев понятия поля гистерезиса ряби намагниченности, действующего на вектор средней намагниченности образца при вращении этого вектора под действием внешнего магнитного поля, приложенного вдоль оси трудного намагничивания (ОТН). Согласно теории ряби намагниченности $\left[{ }^{1}\right]$, неоднородность структуры пленки приводит к неоднородности структуры намагниченности. При этом локальные векторы намагниченности не следят за локальными осями анизотропии, а вследствие обменного и магнитостатического взаимодействий и под действием однородной составляющей поля наведенной анизотропии и внешнего поля образуют определенную магнитную структуру, напоминающую в некоторых случаях рябь на поверхности воды [2]. Мы будем использовать понятие «рябь» намагниченности в более широком смысле, подразумевая под этим термином всякую упорядоченную двумерную магнитную структуру. Согласно теории ряби $[1,3]$, вектор намагниченности магнитной пленки всегда лежит в плоскости пленки и при этом образуются области (субдомены или полуволны ряби) с однородной намагниченностью, разделенные малоугловыми стенками ряби. Допустим, что в исходном состоянии вектор средней намагниченности пленки направлен вдоль оси легкого намагничивания (ОЛН) наведенной анизотропии, а стенки ряби перпендикулярны ОЛН. Если внешнее магнитное поле приложить вдоль ОТН, то происходит процесс вращения вектора средней намагниченности к ОТН. При этом, согласно однодоменной модели Стонера-Вольфарта [ $\left.{ }^{4}\right]$, конечное положение этого вектора определяется на основе уравнения свободной энергии пленки

$$
E=-\mathbf{H} \mathbf{M}=-H^{d} M_{S} \sin \varphi_{0 S-W}+\frac{1}{2} H_{k} M_{S} \sin ^{2} \varphi_{0 S-W}
$$


из условия равновесия

$$
T=\frac{\partial E}{\partial \varphi_{0}}=-H^{d} M_{S} \cos \varphi_{0 S-W}+\frac{1}{2} H_{k} M_{S} \sin 2 \varphi_{0 S-W}=0,
$$

где $T$ - вращающий момент, действующий на среднюю намагниченность; $H^{d}$ - поле вдоль ОТН; $H_{k}$ - поле наведенной анизотропии; फos-w - угол между средней намагниченностью и ОЛН; $M_{S}$ - намагниченность насыщения.

Әксперименты $\left[{ }^{5}\right]$, однако, показывают, что в действительности вектор средней намагниченности пленки поворачивается на угол $\varphi_{0}<\varphi_{0}-W$, причем отставание от положения, предсказываемого однодоменной моделью, растет с увеличением угловой дисперсии намагниченности. Такое отставание можно объяснить взаимодействнем средней намагниченности с полями рассеяния на малоугловых стенках ряби $\left[{ }^{6}\right]$. Как показано в $\left[{ }^{7}\right]$, при определенном значении эффективного поля $\left[{ }^{8}\right]$ происходит т. н. блокировка стенок ряби, т. е. локальное эффективное поле в стенках становится равным нулю. Чем больше дисперсия легких осей, тем больше значение поля блокировки. Можно показать, что у большинства пленок с неразрушающим считыванием уже в отсутствии внешнего поля структура ряби заблокирована и не разблокируется при увеличении внешнего поля вдоль ОТН от нуля до $H^{d}=2-3 H_{k}$. Рассмотрим подробнее, как протекает этот процесс.

При намагничивании ЦТМП вдоль ОТН вектор средней намагниченности вначале поворачивается на некоторый угол так, что стенки ряби остаются неподвижными. Это вращение обратимо. Когда угол $\varepsilon$ между вектором средней намагниченности и нормалью стенок достигает определенного значения, рябь разблокируется сильными поперечными полями размагничивания и при $\varepsilon \neq 0$ нормаль стенок $\mathrm{n}_{0}$ начинает вращаться вслед за средней намагниченностью. При этом на вектор средней намагниченности $\left\langle\mathbf{m}_{1}\right\rangle$ действует тормозящий момент, который можно охарактеризовать т. н. полем гистерезиса ряби $\mathbf{H}_{\varepsilon}$ (см. рис. 1), направленным к $\left\langle\mathbf{m}_{1}\right\rangle$

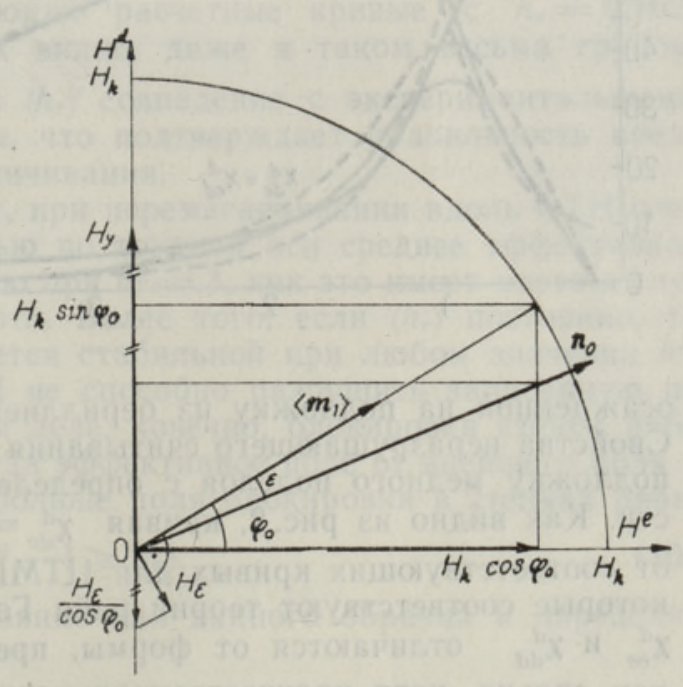

Рис. 1. Диаграмма магнитных полей в уравнении (3). перпендикулярно. Уравнение равновесия (2) принимает тогда вид

$$
T=M_{S}\left(-H^{d} \cos \varphi_{0}+\frac{1}{2} H_{h} \sin 2 \varphi_{0}+H_{\varepsilon}\right)=0,
$$

откуда после приведения всех полей к $H_{k}$ получаем

$$
h^{d}=\frac{H^{d}}{H_{k}}=\sin \varphi_{0}+\frac{h_{\varepsilon}}{\cos \varphi_{0}} .
$$


Условие (4) теперь определяет взаимосвязь между $h^{d}$ и гистерезиса ряби. Поле $h_{\varepsilon}$ в общем случае является функцией от $h^{d}$.

Экспериментальное изучение явления гистерезиса ряби проводилось на установке для снятия кривых дифференциальной магнитной восприимчивости $\left[{ }^{9}\right]$. Пробное поле $H_{\sim}<10^{-3}$ э, его частота 175 кәц, кривые записывались на двухкоординатном самописце. Изучались все четыре компонента тензара восприимчивости $\chi_{d d}^{d}, \chi_{d e}^{d}, \chi_{e d}^{d}$ и $\chi_{e e}^{d}$. Здесь первый нижний индекс обозначает направление пробного поля, второй нижний индекс - направление снятия сигнала, верхний индекс $d-$ перемагничивание вдоль ОТН. Как и следовало ожидать, вследствие симметричности тензора восприимчивости одноосноанизотропного материала $\chi_{d e}^{d}=\chi_{e d}^{d}$. На рис. 2 изображены экспериментальные кривые восприимчивости для однослойной немагнитострикционной пермаллоевой пленки толщиной 0,8 мкм с $H_{k}=4,6$ э, $H_{c}=2,7$ э, $\quad H_{c}^{d}=0,75$ э,

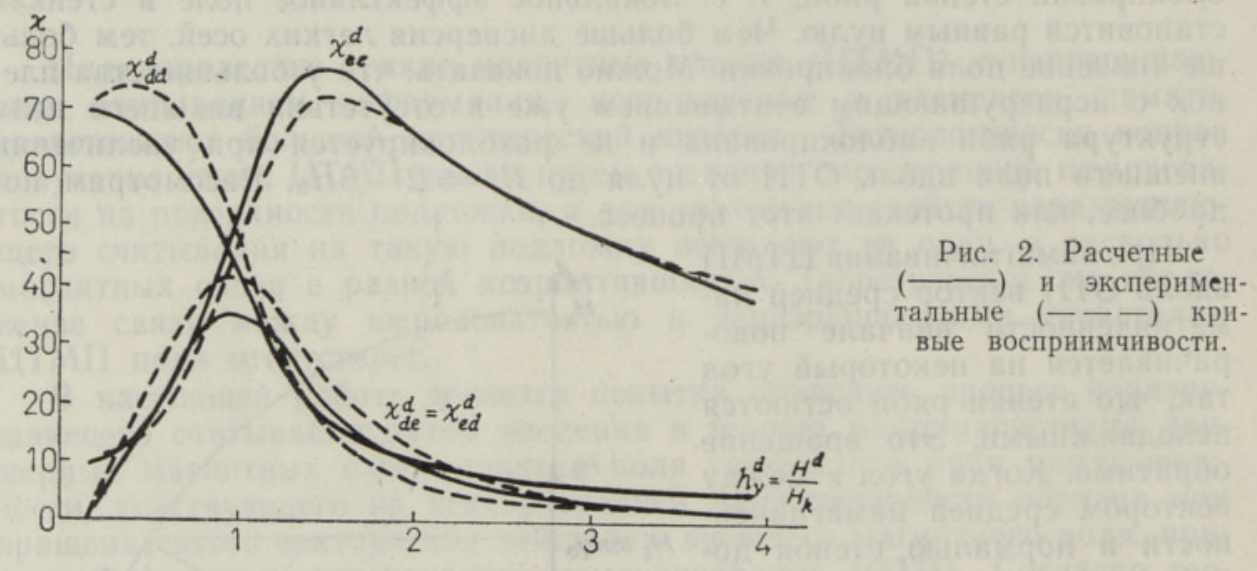

осажденной на подложку из бериллиевой бронзы диаметром 0,15 мм. Свойства неразрушающего считывания достигнуты путем осаждения на подложку медного подслоя с определенной шероховатостью поверхности. Как видно из рис. 2 , кривая $\chi_{d e}^{d}=\chi_{e d}^{d}$ принципиально отличается от соответствующих кривых для ЦТМП с разрушающим считыванием, которые соответствуют теории ряби Гоффмана. Остальные две кривые $\chi_{e e}^{d}$ и $\chi_{d d}^{d}$ отличаются от формы, предсказываемой теорией ряби, не так сильно, хотя количественная разница между линейной теорией и экспериментом и тут весьма существенна.

Покажем теперь, что введенное выше понятие поля гистерезиса ряби позволяет теоретически правильно объяснить форму кривых на рис. 2. Согласно $\left[{ }^{8}\right]$, тензор восприимчивости при $H^{d}>H_{k}$ в общем случае имеет вид

$$
\mathrm{X}=\left\{\begin{array}{c}
\frac{\sin ^{2} \varphi_{0}+\left\langle m_{2}^{2}\right\rangle \cos ^{2} \varphi_{0}}{\left\langle h_{e f f}\right\rangle} \frac{-\sin \varphi_{0} \cos \varphi_{0}+\left\langle m_{2}^{2}\right\rangle}{\left\langle h_{e f f}\right\rangle} \\
\frac{-\sin \varphi_{0} \cos \varphi_{0}+\left\langle m_{2}^{2}\right\rangle}{\left\langle h_{e f f}\right\rangle} \frac{\cos ^{2} \varphi_{0}+\left\langle m_{2}^{2}\right\rangle \sin ^{2} \varphi_{0}}{\left\langle h_{e f f}\right\rangle}
\end{array}\right\},
$$


где $\left\langle m_{2}^{2}\right\rangle-$ среднеквадратичная дисперсия намагниченности, $\left\langle h_{e f f}\right\rangle-$ среднее по образцу эффективное поле.

На основе (3) находим

$$
\frac{\partial^{2} E}{\partial \varphi_{0}^{2}}=\frac{\partial T}{\partial \varphi_{0}}=\frac{M_{S}}{H_{h}}\left(h^{d} \sin \varphi_{0}+\cos 2 \varphi_{0}\right) .
$$

Подставляя сюда $h^{d}$ из (4) получаем

$$
\frac{\partial^{2} E}{\partial \varphi_{0}^{2}}=\frac{M_{S}}{H_{h}}\left(\sin ^{2} \varphi_{0}+\frac{h_{\varepsilon} \sin \varphi_{0}}{\cos \varphi_{0}}+\cos 2 \varphi_{0}\right),
$$

откуда

$$
h_{e f f}=\cos ^{2} \varphi_{0}+h_{\varepsilon} \operatorname{tg} \varphi_{0} .
$$

K выражению (8), согласно $\left[{ }^{8}\right]$, необходимо добавить еще продольное поле рассеяния $\left\langle h_{r}\right\rangle$, так что окончательное выражение для эффективного поля принимает вид

$$
\left\langle h_{e f f}\right\rangle=\cos ^{2} \varphi_{0}+h_{\varepsilon} \operatorname{tg} \varphi_{0}^{\prime}+\left\langle h_{r}\right\rangle .
$$

Подставляя это выражение в (5), находим выражения для всех составляющих тензора восприимчивости с учетом гистерезиса ряби. На рис. 2 приведены соответствующие расчетные кривые с $h_{\varepsilon}=0,162$, $\langle h\rangle_{r}=1,36$ и $\left\langle m_{2}^{2}\right\rangle=0,148$. Как видно, даже в таком весьма грубом приближении постоянных $h_{\varepsilon}$ и $\left\langle h_{r}\right\rangle$ совпадение с экспериментальными кривыми оказывается хорошим, что подтверждает правильность предлагаемого механизма перемагничивания.

Как видно из уравнения (9), при перемагничивании вдоль ОТН пленок с заметной коэрцитивностью по трудной оси среднее эффективное поле не становится равным нулю при $h^{d}=1$, как это имеет место в случае модели Стонера-Вольфарта. Более того, если $\left\langle h_{r}\right\rangle$ постоянно, то средняя намагниченность остается стабильной при любом значении $h^{d}$, т. е. внешнее поле вдоль ОТН не способно разрушить записанную на пленке информацию. На самом деле, конечно, блокировка стенок ряби и вместе с тем $h_{\varepsilon}$ исчезают, когда эффективное поле от внешнего поля и поля анизотропии становятся больше поля блокировки в стенках ряби:

$$
h^{d}-1 \geqslant\left\langle h_{a}\right\rangle \text {, }
$$

где поле блокировки $\left\langle h_{a}\right\rangle$ постоянно для данного образца и определяется, например, согласно $\left[{ }^{7}\right]$

$$
\left\langle h_{a}\right\rangle=\left[\frac{1}{4 \pi \sqrt{2}}\left(\frac{K D \sigma_{1}^{2}}{\sqrt{n}}\right)^{2} M_{S} \sqrt{\bar{d}}\right]^{4 / s} \frac{1}{A K_{u}} .
$$

Здесь $K$ - постоянная локальной анизотропии; $D-$ диаметр неоднородностей структуры с константой анизотропии $K ; \sigma_{1} \approx 0,5 ; n-$ число неоднородностей по толщине пленки; $d$ - толщина пленки; $A$ - постоянная обменного взаимодействия; $K_{u}$ - постоянная наведенной анизотропии.

\section{ЛИТ ЕР АТ У Р А}

1. H a rte K. J., J. Appl. Phys., 39, 1503 (1968).

2. Fuller H. W., Hale M. E., J. Appl. Phys., 31, 239 (1960).

3. Hof $f \mathrm{~m}$ a $\mathrm{n} \mathrm{H}$., IEEE Trans. Magnetics, MAG-4, 32 (1968). 
4. St oner E. C., Wohlf a rth E. P., Phil. Trans. Roy. Soc. (London), A240, 599 (1948).

5. Feldtkeller E., Elektron. Rechenanlagen, 3, 167 (1961).

6. H of $\mathrm{fm}$ a n n H., Ok on M., IEEE Trans. Magnetics, MAG-6, 631 (1970).

7. H of $\mathrm{fm}$ a $\mathrm{n} \mathrm{n}$ H., IEEE Trans. Magnetics, MAG-2, 566 (1966).

8. H of $\mathrm{fm}$ a n n H., Phys. Stat. Sol., 33, 175 (1969).

9. В а лл а сте Х., Изв. АН ЭССР, Физ. Матем., 25, 212 (1976).

Ннститут кибернетики

Академии наук Эстонской ССР
Поступила в редакцию 17/XI 1975

H. VALLASTE

\section{SILINDRILISTE MAGNETKILEDE DIFERENTSIAALSE MAGNETILISE VASTUVOTLLIKKUSE UURIMINE}

Magnetiliste momentide tasakaaluvõrrandile uue liikme - kurdumise hüstereesivälja $h_{\varepsilon}$ - lisamise teel on saadud vōrrand, mis võimaldab kirjeldada diferentsiaalset vastuvôtlikkust eelmagneetimisvälja igasuguste väärtuste korral. Senine teooria oli kasutatav ainult küllastuspiirkonnas.

\section{H. VALLASTE}

\section{ABOUT DIFFERENTIAL MAGNETIC SUSCEPTIBILITY OF PLATED WIRE UNDER HARD AXIS BIAS}

A new approach to susceptibility calculation is proposed. The so-called "ripple hysteresis field" $h_{\varepsilon}$ is added to the magnetic torque equation that enables us to use a single equation for the calculation of differential susceptibility at any value of bias field, not only in the saturation region as in the case of previous ripple theories. 